\title{
Residential Energy Communities: How to minimize the investment risk from an investor perspective David Vangulick ${ }^{1}$, Miguel Manuel de Villena ${ }^{2}$,Raphaël Fonteneau ${ }^{2}$, Damien Ernst ${ }^{2}$ Corresponding author: David Vangulick ${ }^{1^{*}}$
}

\author{
${ }^{1}$ University of Liège, Liège, Belgium and ORES, Gosselies, Belgium \\ ${ }^{2}$ University of Liège, Liège, Belgium \\ *DVangulick@.doct.uliege.be
}

Keywords: Bayesian Game, Distribution System Operator, Grid tariffs, Residential Energy Community

\begin{abstract}
The success of local renewable energy communities, now foreseen by new the European Union directives but also growing worldwide, will rely on the appetite of consumers and investors. This is not obvious when the target local area is a residential community where people have varying expectations. Based on Bayesian game theory (also called game of incomplete information), the purpose of this paper is to define an approach for determining, from the point of view of the renewable energy investor, the level of production capacity and optimum energy price to be offered to the consumers.
\end{abstract}

\section{Introduction}

On December, 18, 2018, the European Parliament and the European Council adopted directive 2018/2001 [1] and introduced the concept of renewable energy communities (RECs). According to this directive, an REC is composed of local consumption and generation of electricity connected to the same network access point (e.g. secondary substation or low-voltage feeder). While the consumption is by local individual consumers, the generation is the result of distributed renewable generation assets, such as solar photovoltaic panels (PVs), which are typically shared across the REC. Under ideal conditions (i.e. depending on the spread between renewable generation technology costs and retail tariff), RECs may decrease electricity prices and thereby lower the size of electricity bills for the participating consumers.

In this paper, we assume: (1), residential consumers are connected to the public distribution system operator's lowvoltage network, fed by the same secondary substation and (2), the investment in distributed renewable generation assets is centralized through an investor.

In [2] the authors show that, for RECs, in addition to simply studying the financial feasibility of the project, it is necessary to acknowledge the local context of the individual consumers (their particular needs) and the way such a context alters the potential benefits and hurdles for the adequate establishment of the project. This context does not makes the investor's decision straightforward. To take into account this behavioural aspect, we introduce several types of consumers, with different rationales behind their decision whether or not to participate in an REC, mimicking the consumer's perception of the benefits brought about by their participation. As for the investor's disposition to create an REC, this will depend on whether the business case makes financial sense.
In addition to these parties, we introduce the distribution system operator (DSO) as an agent who reacts to the deployment of distributed generation by adjusting the distribution tariff to ensure its financial stability (see [3]). This distribution tariff adjustment, in turn, may alter the financial balance of investors and consumers, thereby creating a risk of instability of the community.

In view of all the above, this paper aims to study the level of installed renewable generation capacity and the electricity price offered by an investor to a group of residential consumers so that they will agree to participate in an REC that is stable over time (in terms of the number of consumers), taking into account both the return on investment for the investor as well as the potentially disruptive effect of the REC on the distribution tariff.

We propose to tackle this problem using game theory, and in particular the Bayesian game framework which is designed for problems where players have incomplete information about other players. To achieve this task, we use the mechanism design, a field of game theory which, following an engineering approach, aims to design incentive mechanisms to achieve the desired goals (stable REC community and acceptable return on investment for the investor), where the players (consumers, investor, and distribution system operator) act rationally.

\section{Rounds, players and other assumptions}

\subsection{Definition of the game and key assumptions}

This problem is formulated as a game between the consumers of an REC, the investor in the REC, and the distribution system operator (DSO) serving the REC. Such a game is designed in rounds of equal duration. Let $\mathcal{R}=\left\{R_{1}, \ldots, R_{X}\right\}$ define the set of rounds with $X \in \mathbb{N}$. Moreover, let us define $\tau$ as the period of time between two consecutive rounds $R_{x}$. We assume 
that all the rounds are of equal duration, $\tau$ is constant. We pose $T=\left\{t_{1}, \ldots, t_{Z}\right\}$ the set of periods corresponding to each interval between round $(Z=X-1)$. At each round $R_{x}$, the players in the game can perform different actions depending on their type (see section 3.1). Several assumptions are necessary to solve the game:(i) The players act rationally knowing their type, which is private information;(ii) The investor has perfect information regarding the load profile of each consumer and the generation profile of the generation assets of the REC *; (iii) The DSO tariff's modification (in $€$ ) is unknown by the investor; however the tariff structure is public (i.e. the investor may infer the DSO tariff evolution); (iv) The game is finite (there is an absolute time-stop of the game); and (v) The strategy spaces, the pay-off functions of consumers and investor, the possible types of consumers, and the prior probability distributions of such types, are all common knowledge.

Finally, for the sake of simplicity, all costs and values are constant (there is no net present value computation). In the following section, players of the game are described.

\subsection{Players}

2.2.1 Consumers: These players are the demand providers of the REC; they pay a sum of money to whoever supplies their energy needs, the investor for the energy coming from the REC and/or the retailer for the energy coming from the wholesale market. Their goals will depend on their type. Let denote $\mathcal{N}=$ $\{1, \ldots, N\} \in \mathbb{N}$ the set of indices representing the consumers, with $N>1$ (if $N \leq 1$ there is no game). We assume that all consumers are connected to the public distribution grid and that they fall into one of these three categories:

- Inelastic: these consumers are very not very concerned by the amount of their electricity bill. Let denote $\mathcal{I} \subseteq \mathcal{N}$ the set of inelastic users. This set contains $=N^{i}$ consumers.

- Elastic: these consumers are aware of the amount of their their electricity bill and may take action to reduce it, therefore they will join an REC provided that it represents an economic advantage. Let denote $\mathcal{C} \subseteq \mathcal{N}$ the set of elastic users that contains $N^{e}$ consumers.

- Green: these consumers value the sustainable resources introduced with an REC and, as such, they will join the community depending on the level of self-consumption. Let denote $\mathcal{G} \subseteq \mathcal{N}$ the set of inelastic users. There are $N^{g}$ consumers in this set.

Finally, $N=N^{i}+N^{e}+N^{g}$.

The consumers have, over the tariffication period $\tau$ a demand measured in $\mathrm{kWh}$ and denoted by $D_{n, \tau}$. Such a demand is potentially split into two: $D_{n, \tau}=\kappa_{n, \tau}+\omega_{n, \tau}$, where $\kappa_{n, \tau}$ represents the demand during period $\tau$ that could be covered by the REC's local generation assets whereas $\omega_{n, \tau}$ is the demand during period $\tau$ that could be met by importing electricity from the main network. The two values will depend on the type and

* The authors acknowledge that this assumption may suffer from privacy concerns. In this paper, we consider that all consumers give their consent to share this information with the investor. installed power of the renewable asset that the investor will have proposed.

Some key assumptions are made regarding the consumers: (i) The number of consumers $N$ does not change throughout the game;(ii) The consumers' category is fixed at the beginning and cannot change, and (iii) The consumers' total demands $D_{n, \tau}$ are constant over the game.

2.2.2 Investor: This player is responsible for creating the REC provided that at least two consumers want to join it. In addition, it deploys the local solar PV generation within the REC. Its goal will be to create a working business model for the REC where the investment is recovered. The generator has the following variables associated: $P$ represents the total PV capacity deployed, and $A_{\tau}$ is the total amount of energy in kWh produced during period $\tau$.

Furthermore, we assume that the generation profile is constant for every round of the simulation, and that the PV capacity cannot be modified throughout the game. Hence, $A_{\tau}$ will also be the same for every round of the game.

2.2.3 DSO: The DSO is the player distributing the electricity to the consumers. This player meters the electricity generated/consumed within the REC and, after every round, computes its cost balance. We assume the DSO must break-even at every round and, to that end, it is entitled to change the tariff. Consequently, at the beginning of each round, this agent may modify the tariff.

\section{Modelling the game}

\subsection{Actions of the Agents}

We call the space of actions $\Omega=\left(\Omega_{N}, \Omega_{i n v}, \Omega_{d}\right)$ where $\Omega_{N}$ is the set of actions of consumers, $\Omega_{i n v}$ is the set of actions of the investor, and $\Omega_{d}$ is the set of actions of the DSO. Every action taken by a consumer $n$ at round $R_{x}$ is named $b_{n, R_{x}} \in \Omega_{n}$, this action is defined as follows: $b_{n, R_{x}}=1$ if $n$ joins the REC and $b_{n, R_{x}}=0$ if they do not.

It is important to note that $\Omega_{N}$ is not a function of the consumer's type (inelastic, elastic, or green) and is computed at every round. Similarly, $b_{i n v, R_{x}} \in \Omega_{i n v}$ represents the actions taken by the investor: $b_{i n v, R_{x}}=1$ if the investor creates the REC, 0 if they do not. Pure strategies $S_{n}$ and $S_{i n v}$ are, respectively, the actions taken by a customer $n$ and by the investor for the $X$ rounds. For instance, if $X=3, S_{i n v}(1 ; 1 ; 1)$ denotes that the investor creates and maintains the REC during the whole game.

\subsection{Market model}

In this paper we consider a market model for all the economic transactions corresponding to energy exchanges occurring within the REC. There are different options for the investor when it comes to creating a market for the REC. However, in our work we consider the most general form of such economic transactions as the power purchase agreement (PPA). 
In a PPA, the investor assigns a share of the locally produced energy to each consumer. This is typically called the key of the consumer, and denoted $y_{n, \tau}$. All consumers in the REC must purchase their assigned share, even if they cannot use it. Then, if a particular consumer does not use the purchased energy, the investor will purchase it back, although at a lower price. In this paper, we will assume that the investor determines each consumer's share based on their relative total energy demand and following Equation (1).

$$
\forall n \in \mathcal{N}, y_{n, \tau}=\frac{D_{n, \tau}}{\sum_{n \in \mathcal{N}} D_{n, \tau}}
$$

It should be noted that, given our assumptions, $y_{n, \tau}$ is constant and thus does not depend on the period $t_{z}$ and is also independent of decisions of any players. Thus, each consumer joining the REC will have a certain proportion of their demand covered by locally generated energy. This share will be paid at a price $\xi \in / \mathrm{kWh}$. If part of this energy is not used, it will be sold back to the investor at $\pi € / \mathrm{kWh}$, where $\pi<\xi$. The investor, in turn, will re-sell this energy to the market at $\eta € / \mathrm{kWh}$. Finally, since in this work all locally generated electricity comes from solar $\mathrm{PV}$, the generated energy can benefit from public support mechanisms, such as green certificates; this is modelled introducing $\nu$ in $€ / \mathrm{kWh}$.

The total amount of energy a consumer will receive during each period $t_{z}$ if they join the REC will be equal to $y_{n, \tau} \cdot A_{\tau}$. In addition, the energy put at the disposal of a consumer joining the REC, but not used by them, is represented by $d_{n, \tau}$, and is computed as: $d_{n, \tau}=y_{n, \tau} \cdot A_{\tau}-\kappa_{n, \tau}$

\subsection{Utility and Pay-off Functions}

The utility function of any particular agent represents the net gain of this agent from choosing one action at round $R_{x}$. As for the pay-off function, this represents the net gain of any agent after applying a trajectory of actions (also called strategies in game theory).

3.3.1 Consumers: Concerning the utility function, after a round $R_{x}$, if consumer $n$ does not join the REC, or if there is no REC at all, its utility function for the period of time $t_{z}$ after this round, defined as $u_{n, t_{z}}$, is equal to zero $\left(u_{n, t_{z}}=0\right)$. However, after a round $R_{x}$, if consumer $n$ joins the REC, and the REC is created, the value of $u_{n, t_{z}}$ will be the difference between the (perceived) value the consumer assigns to be part of the REC, and the value remaining outside this community. The value assigned by the consumer to their participation in an REC will depend on their category (see Section 2.2).

To facilitate the resolution of the game, we can monetise the perception of the value (approaching the notion of willingness to pay). A positive $u_{n, t_{z}}$ shows the interest for a consumer $n$ to participate to the REC. It is then possible to state the utility of one consumer $n$ after the period $t_{z}$ as follows (unit: $€): u_{n, t_{z}}=I N_{n, t_{z}}-O U T_{n, t_{z}}$ where $I N_{n, t_{z}}$ is the value of being in the REC, and $O U T_{n, t_{z}}$ is the value of being outside the REC.
$O U T_{n, t_{z}}$ can be easily computed following Equation (2):

$$
O U T_{n, t_{z}}=\mathbf{C}_{c, t_{z}} \cdot \mathbf{V}_{n, \tau}
$$

with $\mathbf{C}_{c, t_{z}}$ being the vector of costs $\mathbf{C}_{c, t_{z}}=\left\langle O_{t_{z}}, P_{t_{z}}, E_{t_{z}}, M_{t_{z}}\right\rangle$ In this vector, $O_{t_{z}}$ is the fixed fee set by the DSO in $€ ; P_{t_{z}}$ is the peak tariff set by the DSO in $€ / \mathrm{kW} ; E_{t_{z}}$ is the energy component of the DSO tariff in $€ / \mathrm{kWh}$; and $M_{t_{z}}$ is the retail market price in $€ / \mathrm{kWh}$. Each of these are set for one simulation period $t_{z}$. Nevertheless, we will consider that the retail market price is a constant from over the simulation period. In addition, $\mathbf{V}_{n, t_{z}}$ represents the vector of volume and quantities $\mathbf{V}_{n, \tau}=\left\langle 1, H_{n, \tau}, D_{n, \tau}, D_{n, \tau}\right\rangle$. In this vector $H_{n, \tau}$ is the peak demand in $\mathrm{kW}$, for consumer $n$ during the period $\tau$, and $D_{n, \tau}$ is the demand, as previously explained.

$I N_{n, t_{z}}$ is expressed as shown in (3):

$$
I N_{n, t_{z}}=\mathbf{C}_{c, t_{z}} \cdot \mathbf{W}_{n, \tau}+\mathbf{C}_{i n v, \tau} \cdot \mathbf{Q}_{n, \tau}+\beta_{n}
$$

where $\mathbf{C}_{c, t_{z}}$ is the same vector as in (2). $\mathbf{W}_{n, \tau}$ is the vector of quantities for the energy still coming from the retail market $\mathbf{W}_{n, \tau}=\left\langle 1, H_{n, \tau}, \omega_{n, \tau}, D_{n, \tau}\right\rangle \cdot \mathbf{C}_{i n v, \tau}$ is the cost vector for the energy coming from the investor $\mathbf{C}_{i n v, \tau}=\left\langle\rho_{\tau}, \psi_{\tau}, \epsilon,-\pi\right\}$ where $\rho_{\tau}$ is the fixed fee to manage the REC (either the DSO or the investor will set up this cost), and $\psi_{\tau}$ is the distribution tariff. $\mathbf{Q}_{n, \tau}$ is the vector and quantities for the energy coming from the investor $\mathbf{Q}_{n, \tau}=\left\langle 1, \kappa_{n, \tau}, y_{n, \tau} \cdot A_{\tau}, d_{n, \tau}\right\rangle$. Finally, $\beta_{n}$ depends on the category of consumer:

$$
\beta_{n}=\left\{\begin{array}{l}
\varsigma ; \forall n \in \mathcal{I} \\
0 ; \forall n \in \mathcal{C} \\
\epsilon_{n} \cdot \kappa_{n, \tau} ; \forall n \in \mathcal{G}
\end{array}\right.
$$

In this equation (4), $\varsigma$ represents a fixed value (in $€$ ) corresponding to the community barrier, which is constant over $\tau$. Finally, $\epsilon_{n}<0$ to reflect the appetite of this type of consumer to be provided with local and green energy.

The actions of the consumers at round $R_{x}$ will thereby be: $b_{n, R_{x}}=1$ if $u_{n, t_{z}}>0$ and 0 if $u_{n, t_{z}} \leq 0$

3.3.2 Investor: In a similar fashion, as we proceeded with the consumers, the utility for the investor will be is zero $\left(u_{i n v, t_{z}}=\right.$ 0 ) if this player decides not to invest on an REC. On the other hand, if the decision to invest is taken, the utility of the investor will be computed as a function of the energy generated and the energy sold to the consumers of the REC.

Each consumer joining the REC will add a value to the utility function of the investor. In particular, the value added by a consumer $n$ joining the REC (which does not depend on the consumer's type) is given by (5)

$$
\operatorname{val}_{n, \tau}=\left(y_{n, \tau} \cdot A_{\tau} \cdot+d_{n, \tau}(\eta-\pi)\right)
$$

The foreseen utility of the investor $\widehat{u}_{i n v, \tau}$ at a given round is computed according to Equation (6):

$$
\widehat{u}_{i n v, \tau}=A_{\tau} \cdot(\nu-R P)+\sum_{n \in \mathcal{N}}\left(v a l_{n, \tau} \cdot b_{n, R_{x}}\right) \quad \forall R_{x} \in \mathcal{R}
$$


where $R P$ represents the reserve price at which the investor could value its generation elsewhere (in $€ / \mathrm{kWh}$ ). A usual value for the reserve price is, for instance, the levelised cost of energy.

As for the pay-off function, it is now possible to formulate the pure strategy pay-off matrix of the investor:

$$
P O_{i n v}=\sum_{\tau \in \mathcal{T}, R_{x} \in \mathcal{R}} \widehat{u}_{i n v, \tau} \cdot b_{i n v, R_{x}}
$$

\section{Mechanism Design}

The main problem for the DSO is anticipating the momentum when externalities (grid costs, taxes, and levies) that are not supported by these community, are so large that they endanger the sustainability of the DSO and/or the general welfare. This is a classic "public goods game" issue. Penalising those who benefit from cooperation without participating (also called "free riders") may provide a solution to this problem. The aim of the penalties is to provide an incentive where cooperation could still pay (e.g., see [4]). To simulate this penalisation, we assume that the DSO's income has to remain stable during the whole economic life cycle of the REC. The DSO's role is defined by the game theory as a "Third-party Punishment", as described in [5].

To compute the revenues of the DSO that should be kept constant, we can make use of the difference between the forecast of the revenue $\left(\widehat{Y_{t_{z}}}\right)$ and the actual revenue $\left(Y_{t_{z}}\right)$. The former is computed taking into account the situation at the simulation period $t_{z-1}$ (consumption, peak, and participation to the REC) while the latter is computed ex-post, from the data collected after the period is finished. Finally, we can define $\Delta Y_{t_{z}}$ as the difference $\widehat{Y_{t_{z}}}-Y_{t_{z}}$. As others papers conclude (e.g. [6]), if the DSO chooses to update (increase) the energy component of the distribution tariff $\left(E_{t, z}\right)$ in order to cover $\Delta Y_{t_{z}}$, this reinforces the interest of consumers to join the community because this tariff increase will only be paid for the energy not coming from the REC (i.e. imports from the main network), and thus mainly affecting non-members of the REC. That is why, in our paper, the DSO will affect $\Delta Y_{t_{z}}$ to an increase of the fixed fee $O_{t_{z}}$.

The objective of the investor is to obtain the maximum payoff upon reaching the end of the simulation periods.

$$
\max P O_{i n v}
$$

To reach this objective, the investor has three decision variables, namely the strategy vector $S_{i n v}$, the installed capacity $P$ and the selling price. The information available for the investor to maximise their utility is listed in table 1 . In addition, the investor knows the reference energy price and an approximation of the proportion of each type of consumer and their values of $\beta_{n}$. Finally the investor also knows the DSO's distribution tariff adjustment.

\section{Solution Technique}

To find the optimum $P O_{i n v}$ shown in Equation 8, we explore the space of solutions by running a Monte-Carlo simulation. Figure 1 describes the algorithm.
Table 1 Information available for the investor.

\begin{tabular}{|c|c|}
\hline Parameter & Meaning \\
\hline RP Price & Reserve price \\
$\nu$ & Public support \\
$\eta$ & Selling price to the market \\
$\pi$ & Purchasing price to the consumers \\
$d_{n, \tau}$ & Demand of each consumer \\
$A_{\tau}$ & Amount of energy produced by the PV \\
\hline
\end{tabular}

Fig. 1. Monte-Carlo simulations.

1. Set the number of simulations;

2. Define the solution space to be explored by setting the boundaries of installed capacity $P$, the selling price $\xi$, and the ratio $\frac{\pi}{\xi}$;

3. Populate all the constants known by the investor (see table 1);

4. Loop until all the investor strategies $S_{i n v}$ have been explored

(a) Step 1: Initialisation: Select one of the investor strategies; $S_{i n v}$ (e.g. $\left.S_{i n v}=(1 ; 1 ; 0)\right)$ and the corresponding reserve price RP.

(b) Step 2: Simulations: loop until number of simulations is reached

(1) Randomly attribute consumer types to a specific consumer: $n \rightarrow$ type (inelastic, elastic, green) taking into account the proportion of consumer types; giving its type, randomly attribute, for each consumer, value of $\beta_{n}$ (taking into account the well-known distribution of these values);

(2) Explore the solution space for every pairs $(P, \xi)$ : For each period $t_{z}(z=1,2, \ldots X)$ and for each customer $n$ :

- Compute $y_{n, \tau} \cdot A_{\tau}$ and $d_{n, \tau}$ using the corresponding load and generation profiles;

- $\quad$ Compute $u_{n, t_{z}}$ using equations (2), (3);

- Compute $v a l_{n, \tau}$ from equation (5);

- Determine the decision of $n$ to join the REC if $u_{n, t_{z}}>0$

- $\quad$ Determine $\widehat{u}_{i n v, t_{z}}$ with equation (6);

- Compute DSO's reaction.

(c) Step 3: Results: Compute $P O_{i n v}$ with equation $(7)$ and find the pair $(P$,$) that has the maximum$ $P O_{\text {inv }}$.

An important aspect of the procedure explained in Figure 1 is the determination of the reserve price. Indeed, if the investor decides to opt for a strategy $S_{i n v}=(1 ; 1 ; 1)$, the reserve price can be computed on the basis that the investment will remain active during the three simulation periods. On the other hand, if the investor opts for a strategy $S_{i n v}=(1 ; 1 ; 0)$, only two periods can be used (i.e., 10 years if $\tau=5$ ). Finally, if the investor opts for a strategy $S_{i n v}=(1 ; 0 ; 0)$, the investment must be profitable over a single period (i.e. 5 years). 
Table 2 Results of simulation for $S_{i n v}=(1 ; 1 ; 1)$

\begin{tabular}{|c|c|c|c|}
\hline Optimal installed capacity & 160 & 170 & 180 \\
\hline Max $P O_{i n v}$ of these simulations & 6799 & 6031 & 4095 \\
\hline Min $P O_{i n v}$ of these simulations & 1588 & 1668 & 1781 \\
\hline Average $P O_{i n v}$ of these simulations & 4653 & 4168 & 3039 \\
\hline
\end{tabular}

\section{Numerical example}

In this section, we propose a numerical example to showcase the performance of the developed simulator. In particular, we run the simulation on a neighbourhood consisting of 20 residential consumers who are not yet equipped with PV panels, with $\tau=5$ years and $X=3$. The metering (generation and load) data are coming from real-world cases of a commercial street even though the consumers are anonymous. Since the focus of this paper is to provide results of the approach, the determination of the proportion of each type of consumer, and the distribution of $\beta_{n}$ and $\epsilon_{n}$ are provided in a complementary note [7]. This note explains how it is possible to calculate these values with a good approximation of these values and it gives the numerical values for the simulations.

From our simulations, we observe that for the strategies $S_{i n v}=(1 ; 1 ; 0)$ and $S_{i n v}=(1 ; 0 ; 0)$, there is no positive $P O_{i n v}$ value. This means that, considering the values selected, the rational investor must always remain in the community. These results indicate that, for these two strategies, the reserve price is too high compared to the utilities that could be drawn from consumers. As a result, only consumers of type green and with their $\xi_{n}$ close to the upper bound will join to the community. However, as soon as the DSO adjusts the distribution tariff, they leave the REC.

The results for the strategy $S_{i n v}=(1 ; 1 ; 1)$ show that the price corresponding to the maximum value is always equal to $0.04 € / \mathrm{kWh}$. The to-be-installed capacity $P$ varies between 160 and $180 \mathrm{~kW}$. More details of these simulations can be found in Table 2. It can be observed that the value of $P=160$ $\mathrm{kW}$ is the one that is the most frequent optimum and leads to the highest average and maximum $P O_{i n v}$ value. The pair $(P=$ $160 \mathrm{~kW}, \xi=0.04 € / \mathrm{kWh}$ ) is therefore the optimal strategy for the investor.

We observe that for the consumer, the surplus of energy creates a negative valuation due to the investor's purchase of this energy at a lower price than the original purchasing price $(\pi<\xi)$. This results in consumers of type elastic to leave the community, making it less attractive for the investor.

Another interesting point to note is that, due to the reaction of the DSO, some consumers of type elastic and green leave the REC after the first round. In our illustrative simulation, 17 out of 20 consumers join the community at the first round while, for round 2 , only 11 remain. The evolution of the expected utility for the investor is for $T 1: 2555, T 2: 1644$, and $T 3: 1644$, for a total of 5843 . The drop in revenue between $T 1$ and $T 2$ is significant but this erosion stops at $T 3$.

\section{Conclusion}

The proposed method can be used by an investor to evaluate the "installed capacity - price" pair, which will have the greatest potential of creating a stable energy community but also meeting the investor's economic expectations. With the simulations based on a real-life Walloon case, it can be shown that the investor has to opt for a long-term strategy. The reaction of the DSO can be integrated into these simulations and has an important effect on the REC. Regarding comments about the presented results, the numerical example considers only 20 residential consumers. In real DSO network this number is generally larger (for instance the average in the Walloon region is up to 80 consumers per distribution substation). The proposed method can be upgraded to this level without any issue. Nevertheless, the assumption about the perfect information relating to load and generation for the investor becomes less and less realistic as the number of people involved increases. As fare as the room for future work is concerned, we have not yet integrated electrical vehicles or residential local storage into our calculation. These two elements may have an important impact on the expected utility for the consumer that could well be worth a further treatment.

\section{References}

[1] European Union. "Directive 2018/2001 of the European Parliament and of the Council of 11 December 2018 on the promotion of the use of energy from renewable sources". In: Official Journal of the European Union 328 (2018), pp. 82-209.

[2] Liam Byrnes, Colin Brown, Liam Wagner, and John Foster. "Reviewing the viability of renewable energy in community electrification: The case of remote Western Australian communities". In: Renewable and Sustainable Energy Reviews 59 (2016), pp. 470-481.

[3] Miguel Manuel de Villena, Raphael Fonteneau, Axel Gautier, and Damien Ernst. "Evaluating the Evolution of Distribution Networks under Different Regulatory Frameworks with Multi-Agent Modelling”. In: Energies 12.7 (2019), p. 1203.

[4] Fehr, E., \& Gachter, S. (2002). Altruistic punishment in humans. Nature, 415(6868), 137.

[5] Fehr, E., \& Fischbacher, U. (2004). Third-party punishment and social norms. Evolution and human behavior, 25(2), 63-8

[6] Abada, I., Ehrenmann, A., \& Lambin, X. (2018). Unintended consequences: The snowball effect of energy communities Available at https://www.repository.cam.ac.uk /bitstream/handle/1810/277393/cwpe1828.pdf?sequence $=1$

[7] David Vangulick University of Liège, Liège, Belgium Residential Energy Communities: Complementary notes at http://hdl.handle.net/2268/239886 